\title{
Desempenho de tratores agrícolas em tração
}

\author{
João Manuel Pereira Ramalho Serrano(1)
} (1)Universidade de Évora, Instituto de Ciências Agrárias Mediterrânicas, Dep. de Engenharia Rural, Núcleo da Mitra, Apartado 94 ,
7002-554 Évora, Portugal. E-mail: jmrs@uevora.pt

\begin{abstract}
Resumo - O objetivo deste trabalho foi avaliar o desempenho de tratores agrícolas e implementos em trabalhos de tração. Procurou-se estabelecer e validar modelos de previsão relativos ao esforço de tração requerido pela grade de discos offset de arrasto e relativos ao consumo de combustível do trator. É descrito o sistema de aquisição de dados desenvolvido para utilização em ensaios de campo, em condições reais de trabalho. Os parâmetros medidos foram: o regime do motor, a velocidade teórica e a velocidade real de avanço, o consumo horário de combustível e a força de tração na barra. Também foram realizados testes dos tratores com um freio dinamométrico ligado à tomada de potência. Os resultados de um projeto de pesquisa de três anos foram usados para estabelecer uma equação de previsão do esforço requerido por grades de discos offset, válida para mobilização primária de solos secos, de textura franca. Nas mesmas condições, os resultados validam a relação linear entre a força de tração por unidade de largura de trabalho do implemento e o consumo de combustível por hectare, indicador do dimensionamento do conjunto trator-implemento. Os dados obtidos também evidenciam as vantagens de se selecionarem marchas altas e baixa rotação do motor.
\end{abstract}

Termos para indexação: mecanização, instrumentação, previsão do esforço, consumo de combustível.

\section{Performance of agricultural tractors in traction}

\begin{abstract}
The objective of this work was to evaluate the performance of agricultural tractors and tillage implements. Also, it was sought to establish mathematical models to estimate the draught requirement of disc harrows, and to validate the relationship between fuel consumption per hectare and the specific drawbar pull. The data acquisition system developed to use in field tests, under real working conditions, permitted the measure of following performance parameters: engine speed, theoretical and actual forward speed, fuel consumption per hour and drawbar pull. Tests were also made with a power take-off dynamometer. The results of a three years research project were used to establish a mathematical model to estimate the draught requirement of the disc harrows, valid for dry, undisturbed loam soils, and to validate the linear relationship between the drawbar pull per unit of implement width and the fuel consumption per hectare, which indicates the tractorimplement dimensioning. The data also put in evidence the benefits of the "gear up, throttle down" approach.
\end{abstract}

Index terms: mechanization, instrumentation, drawbar pull prediction, fuel consumption.

\section{Introdução}

O progresso no setor da Metrologia Aplicada, ocorrido depois de 1960, ligado ao desenvolvimento de sensores e da própria informática, com a miniaturização dos sensores e dos componentes eletrônicos, tornou possível a aplicação de transdutores no trator, sem necessidade de introduzir modificações geométrico-mecânicas em sua estrutura (Ragni \& Santoro, 1997).

Um sistema de medição de parâmetros físicos do desempenho de tratores agrícolas depende, fundamentalmente, do tipo de sensores que o constitui. Os sistemas mais habituais, de concepção relativamente simples, normalmente de custo reduzido, e desenvolvidos de forma a permitir fácil adaptação a qualquer trator em condições de trabalho muito diversas, têm como sensores: um radar, uma célula de carga, um medidor de fluxo e sensores magnéticos de proximidade. Alguns exemplos desses modelos são apresentados por Bowers (1985), Riethmuller (1989), Peça et al. (1998), Mantovani et al. (1999) e Al-Janobi (2000).

A estrutura de um sistema de aquisição de dados (SAD) inclui, para além dos sensores, uma unidade de tratamento e condicionamento de sinal (conversão de sinais analógicos em sinais digitais), ligada por interface (placa de aquisição de dados) a um sistema de armazenamento e tratamento dos dados (computador). Podem ser encontradas referências a sistemas 
perfeitamente autônomos e versáteis, construídos de raiz e adaptáveis a qualquer trator agrícola. Outra possibilidade consiste em aproveitar parte da instrumentação eletrônica, que equipa comercialmente alguns modelos de tratores agrícolas modernos (sistemas de informação ao operador), adaptando-se instrumentação complementar necessária para medição de outros parâmetros e para o registro e armazenamento de dados que habitualmente não são registrados. Este segundo modelo exige, tal como o primeiro, a calibração dos sensores e apresenta a grande vantagem de aproveitar o potencial existente, constituindo-se como uma estrutura simples e de custo relativamente baixo.

A análise das referências bibliográficas, citadas na literatura, revela a publicação de trabalhos de instrumentação e acompanhamento de tratores agrícolas desde os anos sessenta até a atualidade, o que demonstra a importância da aquisição de dados na correta gestão do conjunto trator-implemento, na comparação de sistemas alternativos de mobilização do solo ou na confirmação de relações teóricas entre variáveis, para efeitos de modelação e de compreensão da complexa dinâmica envolvida: trator-implemento-solo. Os principais aspectos que as equipes procuram investigar são: a determinação da força de tração requerida pelos implementos de mobilização do solo (Glancey et al., 1996; Al-Suhaibani \& Al-Janobi, 1997; Al-Janobi \& Al-Suhaibani, 1998), e a energia envolvida nos diferentes sistemas de preparação do solo, para instalação das culturas (Bowers, 1985; Riethmuller, 1989; Perfect et al., 1997; Serrano et al., 2003). A base de dados criada nesses estudos permite $o$ apoio à tomada de decisão do agricultor, no que se refere ao dimensionamento do conjunto trator-implemento, e a avaliação global do desempenho do trator. A avaliação energética pode ser realizada com base na medição do consumo de combustível por hectare, principal indicador técnico de referência na avaliação da eficiência de utilização do trator agrícola, uma vez que traduz o envolvimento das diversas variáveis que condicionam o rendimento global da transformação do combustível fornecido ao motor em trabalho útil realizado pelo implemento.

Em explorações de sequeiro do Sul de Portugal, as grades de discos são muito populares, utilizadas como implementos de mobilização primária e mobilização secundária, em rotações de três anos, constituídas por: cereal de inverno/cereal de inverno/girassol (Serrano et al., 2003). Contudo, em Portugal, a ausência de dados leva a que as decisões de dimensionamento do conjunto trator-implemento sejam tomadas com base em conhecimentos empíricos. Os tratores são normalmente selecionados de acordo com as necessidades de potência dos implementos usados em mobilização primária, como arados de discos e subsoladores, o que conduz freqüentemente ao sobredimensionamento do trator, em relação aos implementos usados em mobilização secundária, como a grade de discos e os cultivadores. Este aspecto é agravado pela tendência de aumento da potência dos tratores. Além disso, é difícil responder as questões relacionadas ao dimensionamento correto, em face da variabilidade das condições do solo.

O objetivo deste trabalho foi avaliar o desempenho de tratores agrícolas e implementos, em trabalhos de tração em condições reais, e estabelecer e validar modelos de previsão do esforço de tração requerido pela grade de discos offset de arrasto e do consumo de combustível do trator.

\section{Material e Métodos}

O trabalho foi realizado no período de 2000 a 2002, em explorações agrícolas dos distritos de Évora e Beja, na Região Sul de Portugal; na Tabela 1 é apresentada a localização dos ensaios.

$\mathrm{Na}$ aquisição de dados, aproveitou-se parte da infraestrutura do sistema de informação comercial Datatronic (que equipa os tratores Massey-Ferguson das séries 3000 e 3100), constituído por um conjunto de sensores ligados à unidade central de tratamento do sinal e de cálculo, que permite ao operador informar-se sobre parâmetros relevantes do desempenho do trator. Os sensores do sistema Datatronic são: um radar Doppler, instalado por baixo do trator; um sensor magnético de proximidade,

Tabela 1. Coordenadas geográficas dos locais de ensaio, na região Sul de Portugal.

\begin{tabular}{|c|c|c|c|c|}
\hline $\begin{array}{l}\text { Código } \\
\text { do local }\end{array}$ & Concelho & $\begin{array}{l}\text { Designação da } \\
\text { propriedade }\end{array}$ & Latitude & Longitude \\
\hline 1 & Selmes & Cortes de Cima & $38^{\circ} 09^{\prime} 06,6^{\prime \prime} \mathrm{N}$ & $7^{\circ} 43^{\prime} 24,2^{\prime \prime} \mathrm{W}$ \\
\hline 2 & Aguiar & Campo da Mira & $38^{\circ} 30^{\prime} 36,8^{\prime \prime N}$ & $7^{\circ} 53^{\prime} 10,0^{\prime \prime} \mathrm{W}$ \\
\hline 3 & Alcáçovas & Tojal & $38^{\circ} 28^{\prime} 00,6^{\prime \prime} \mathrm{N}$ & $8^{\circ} 01^{\prime} 02,8^{\prime \prime} \mathrm{W}$ \\
\hline 4 & Portel & Outeiro & $38^{\circ} 19^{\prime} 07,6^{\prime \prime N}$ & $7^{\circ} 53^{\prime} 58,6^{\prime \prime} \mathrm{W}$ \\
\hline 5 & Arraiolos & Oliveiras & $38^{\circ} 43^{\prime} 06,5^{\prime \prime} \mathrm{N}$ & $8^{\circ} 00^{\prime} 14,3^{\prime \prime} \mathrm{W}$ \\
\hline 6 & Oriola & Lentisca-a & $38^{\circ} 21^{\prime} 18,2^{\prime \prime} \mathrm{N}$ & $7^{\circ} 54^{\prime} 33,2^{\prime \prime} \mathrm{W}$ \\
\hline 7 & Oriola & Len & $38^{\circ} 21^{\prime} 09,2^{\prime \prime} \mathrm{N}$ & $7^{\circ} 54^{\prime} 15,6^{\prime \prime} \mathrm{W}$ \\
\hline 8 & Valverde & $\mathrm{Caba}$ & $38^{\circ} 32^{\prime} 16,9^{\prime \prime} \mathrm{N}$ & $8^{\circ} 01^{\prime} 26,6^{\prime \prime} \mathrm{W}$ \\
\hline 9 & Évora & Louseiro & $38^{\circ} 31^{\prime} 41,4^{\prime \prime} \mathrm{N}$ & $7^{\circ} 48^{\prime} 19,3^{\prime \prime} \mathrm{W}$ \\
\hline 10 & Monsaraz & Revilheira & $38^{\circ} 27^{\prime} 51,6^{\prime \prime} \mathrm{N}$ & $7^{\circ} 28^{\prime} 45,1^{\prime \prime} \mathrm{W}$ \\
\hline 11 & Oriola & Vale Figueira & $38^{\circ} 19^{\prime} 56,3^{\prime \prime} \mathrm{N}$ & $7^{\circ} 53^{\prime} 22,7^{\prime \prime} \mathrm{W}$ \\
\hline
\end{tabular}


colocado junto ao volante do motor; um sensor magnético de proximidade, colocado junto ao grupo cônico da transmissão às rodas traseiras; um medidor de fluxo, instalado no sistema de alimentação de combustível; e um sensor de posição, colocado nos braços de elevação do sistema hidráulico.

Para superar o fato de a informação não ficar registrada, foi desenvolvido um sistema de aquisição de dados (SAD). A instalação de uma tomada em $\mathrm{T}$, na ligação entre o computador e o painel do sistema de informação, permitiu derivar os sinais dos sensores referidos para um circuito eletrônico constituído por: uma caixa de terminais (com oito canais) e de condicionamento de sinal, para converter sinais analógicos registrados pelos sensores em sinais digitais; uma placa de aquisição de dados, como interface para um computador portátil, sistema de armazenamento e tratamento de dados (Serrano, 2002). Além dos sensores referidos, foi instalada uma célula de carga, interposta na ligação trator-implemento.

O computador portátil IBM Compaq Armada 1520, a placa de aquisição de dados do tipo PCMCIA, modelo DAQCard-1200 da National Instruments, a tomada de derivação de sinais do sistema Datatronic, a célula de carga UBM U2A, de $50 \mathrm{kN}$ de carga máxima, e todos os cabos de ligação que constituem o sistema de aquisição de dados, instalado a bordo do trator durante os ensaios, são equipamentos comerciais disponíveis no mercado.

A autonomia do SAD, durante a coleta de dados no campo, é de cerca de 4 horas consecutivas, garantida por duas baterias interiores recarregáveis por ligação à corrente elétrica. Foi ainda adaptado um cabo de ligação do sistema à bateria do trator, para permitir o registro dos dados em ensaios mais prolongados.

Uma aplicação Labview foi desenvolvida e instalada no computador portátil para o controle do processo de aquisição, tratamento e armazenamento de dados, durante os trajetos dos ensaios.

As informações do regime do motor $\left(\mathrm{min}^{-1}\right)$, da velocidade teórica de avanço $\left(\mathrm{km} \mathrm{h}^{-1}\right)$, da velocidade real $\left(\mathrm{km} \mathrm{h}^{-1}\right)$, da força de tração na barra $(\mathrm{N})$ e do consumo horário $\left(\mathrm{L} \mathrm{h}^{-1}\right)$ foram registradas em intervalos de um segundo, tendo-se interrompido o registro no final de cada trajeto operativo, pela atuação manual do interruptor de posição de trabalho, do painel de comando do sistema hidráulico do trator. Os dados destas cinco funções, correspondentes a cada trajeto operativo, realizado pelo conjunto trator-grade de discos, constituíram um fichário identificado com um código data-hora, automaticamente definido no programa do computador portátil.

Ainda, para a aquisição de dados, foi utilizado um freio dinamométrico, modelo Tractor Test Centre XT200, equipado com um medidor de momento e um sensor magnético de proximidade para leitura do momento e do regime à tomada de potência. Esses dados, com a informação do medidor de fluxo de combustível do trator, foram registrados e armazenados em um computador portátil. Os resultados foram tratados no programa Surfer, o qual, pelos processos de triangulação e de interpolação linear, estabeleceu as curvas de iso-consumo do motor. Essas curvas de desempenho representam uma autêntica radiografia do rendimento potencial do motor. A partir desta informação é possível aferir a utilização de um trator agrícola em diferentes operações culturais, e buscar sempre a maior eficiência de conversão do combustível em energia mecânica.

A instrumentação descrita permitiu a coleta de dados dos ensaios de campo, de mobilização primária de diferentes tipos de solo, tendo sido utilizados equipamentos que incluíram tratores de $59 \mathrm{~kW}$ a $134 \mathrm{~kW}$ de potência máxima no motor e grades de discos offset de 2 a $4 \mathrm{~m}$ de largura de trabalho, com massa de 1.200 a $3.500 \mathrm{~kg}$ (Tabela 2). Foram testadas, em cada local, duas situações de abertura dos corpos da grade de discos, consideradas pelo agricultor como adequadas para a realização do trabalho pretendido. Foram, também, testadas duas combinações de regime de funcionamento do motor: uma correspondente à seleção do regime nominal do motor (para otimização da capacidade de trabalho) e outra correspondente à seleção de um regime de cerca de $80 \%$ do regime nominal (para otimização do consumo de combustível por hectare), ambas combinadas com a seleção da relação de transmissão mais alta permitida.

Em cada local e para cada regulagem tratorimplemento, foram realizadas quatro repetições de trajetos operativos, com cerca de 80 a 100 m de extensão, tendo sido medidos os parâmetros: abertura dos corpos da grade ( $\alpha$, em graus), largura de trabalho (l, em m) e profundidade de trabalho (d, em $\mathrm{m}$ ), e recolhidas amostras de solo até $20 \mathrm{~cm}$ de profundidade, para determinação da textura e do teor de umidade. Em cada local, previamente aos ensaios para registro de dados, foram realizados testes para estabelecimento das regulagens, nas grades de discos (abertura dos corpos) e no trator (regime de rotação do motor e relação de transmissão). 


\section{Resultados e Discussão}

Os ensaios de campo, realizados em situações de mobilização primária de solos de textura média, permitiram estabelecer um modelo de previsão da força de tração na barra (T, em N) em função da massa da grade de discos (m, em $\mathrm{kg}$ ), a qual se pode expressar matematicamente pela equação $\mathrm{T}=7,3965 \mathrm{~m}+7541,6$, com coeficiente de determinação de 0,79. Este modelo de previsão confirma o modelo apresentado pela ASAE (1995) para grades de discos offset, em mobilização primária de solo de textura média pela equação $\mathrm{T}=11,7 \mathrm{~m}$.

A Figura 1 enquadra os valores de força de tração medidos $\left(\mathrm{T}_{\mathrm{med}}\right)$ nos ensaios de campo, no modelo de previsão da ASAE (força de tração prevista, $\mathrm{T}_{\text {prev }}$ ). Este modelo ASAE, que se baseava na previsão da força de tração a partir da massa da grade e da textura do solo, era um modelo redutor, que não considerava o diâmetro dos discos, o ângulo de abertura dos corpos da grade, a profundidade e a velocidade de trabalho. Em 2000 (ASAE, 2000), foi apresentada uma revisão da equação de previsão do esforço de tração requerido por grades de discos, com a inclusão da largura, da velocidade e da profundidade de trabalho. Esta proposta apresentava, contudo, uma condicionante importante ao exigir o conhecimento da profundidade de trabalho, parâmetro não previsível em implementos de arrasto desprovidos de rodas reguladoras da profundidade. O modelo ASAE (1995) é, por isso, de aplicação mais fácil e aceitável para efeitos de dimensionamento do conjunto tratorimplemento, comprovado pelos resultados obtidos.

A medição dos parâmetros tração na barra (T), velocidade real de avanço $\left(\mathrm{v}_{\mathrm{r}}\right)$ e consumo horário de combustível (Ch), nos ensaios de campo, comparou-se

Tabela 2. Características dos tratores, das grades de discos offset e dos solos, nos locais de ensaio.

\begin{tabular}{|c|c|c|c|c|c|c|c|c|c|c|}
\hline \multirow[t]{2}{*}{ Local } & \multirow{2}{*}{$\begin{array}{l}\text { Trator } \\
\text { (potência } \\
\text { máxima, kW) }\end{array}$} & \multicolumn{5}{|c|}{ Grade de discos $^{(1)}$} & \multicolumn{4}{|c|}{ Solo } \\
\hline & & $\begin{array}{c}\text { Marca e modelo } \\
\text { ( } \mathrm{n}^{\mathrm{o}} \text { de discos }- \text { diâmetro dos } \\
\text { discos })\end{array}$ & $\begin{array}{c}\mathrm{m} \\
(\mathrm{kg})\end{array}$ & $\begin{array}{c}\alpha \\
\left(^{\circ}\right)\end{array}$ & $\begin{array}{c}1 \\
(\mathrm{~m})\end{array}$ & $\begin{array}{c}\mathrm{d} \\
(\mathrm{m})\end{array}$ & $\begin{array}{c}\text { Areia } \\
\left(\mathrm{g} \mathrm{kg}^{-1}\right)\end{array}$ & $\begin{array}{c}\text { Limo } \\
\left(\mathrm{g} \mathrm{kg}^{-1}\right)\end{array}$ & $\begin{array}{l}\text { Argila } \\
\left(\mathrm{g} \mathrm{kg}^{-1}\right)\end{array}$ & $\begin{array}{l}\text { Umidade } \\
\text { relativa }(\%)\end{array}$ \\
\hline \multirow[t]{2}{*}{1} & MF3680 (134) & Galucho (GLHR36-26") & 3.500 & 25 & 3,95 & 0,085 & 480 & 230 & 290 & 4,0 \\
\hline & & & & 34 & 3,95 & 0,105 & & & & \\
\hline \multirow[t]{2}{*}{2} & MF3095 (81) & Galucho (GLHR24-26") & 1.870 & 53 & 2,93 & 0,180 & & & & \\
\hline & & & & 31 & 2,93 & 0,180 & 680 & 130 & 190 & 11,5 \\
\hline \multirow[t]{2}{*}{2} & MF3060 (59) & Herculano (HPR20-24") & 1.300 & 46 & 2,07 & 0,180 & & & & \\
\hline & & & & 37 & 2,10 & 0,180 & & & & \\
\hline \multirow[t]{2}{*}{3} & MF3095 (81) & Premetal (PLHR26-26") & 2.700 & 44 & 3,01 & 0,180 & & & & \\
\hline & & & & 33 & 3,13 & 0,150 & 730 & 90 & 180 & 15,0 \\
\hline \multirow[t]{2}{*}{3} & MF3060 (59) & Herculano (HPR20-24") & 1.300 & 46 & 2,06 & 0,180 & & & & \\
\hline & & & & 37 & 2,13 & 0,166 & & & & \\
\hline 4 & MF3650 (110) & Galucho (GSM24-28") & 2.000 & 44 & 2,89 & 0,180 & 490 & 230 & 280 & 12,0 \\
\hline 4 & MF3060 (59) & Herculano (HPR20-24") & 1.300 & 46 & 2,08 & 0,190 & & & & \\
\hline \multirow[t]{2}{*}{5} & MF8130 (114) & Premetal (PLHR26-26") & 2.700 & 43 & 3,19 & 0,180 & & & & \\
\hline & & & & 27 & 3,31 & 0,180 & 730 & 100 & 170 & 19,0 \\
\hline \multirow[t]{2}{*}{5} & MF3060 (59) & Herculano (HPR20-24") & 1.300 & 46 & 2,17 & 0,165 & & & & \\
\hline & & & & 37 & 2,10 & 0,165 & & & & \\
\hline 6 & MF3095 (81) & Galucho (A2CP24-26") & 1.460 & 54 & 2,43 & 0,145 & & & & \\
\hline \multirow[t]{2}{*}{6} & MF3095 (81) & Halcon (28-24") & 1.650 & 43 & 3,30 & 0,132 & & & & \\
\hline & & & & 37 & 3,36 & 0,156 & 690 & 130 & 180 & 8,0 \\
\hline \multirow[t]{2}{*}{6} & MF3060 (59) & Herculano (HPR20-24") & 1.300 & 46 & 2,07 & 0,182 & & & & \\
\hline & & & & 37 & 2,11 & 0,158 & & & & \\
\hline \multirow[t]{2}{*}{7} & MF3060 (59) & Galucho (A2CP24-26") & 1.460 & 54 & 2,52 & 0,170 & & & & \\
\hline & & & & 38 & 2,60 & 0,130 & 650 & 100 & 250 & 8,0 \\
\hline 7 & MF3060 (59) & Galucho (A2CP22-24") & 1.180 & 54 & 2,20 & 0,140 & & & & 8,0 \\
\hline 7 & MF3060 (59) & Herculano (HPR20-24") & 1.300 & 46 & 2,08 & 0,190 & & & & \\
\hline 8 & MF3060 (59) & Herculano (HPR20-24") & 1.300 & 46 & 2,18 & 0,170 & 750 & 90 & 160 & 8,0 \\
\hline 9 & MF3060 (59) & Herculano (HPR20-24") & 1.300 & 46 & 2,09 & 0,220 & 640 & 200 & 160 & 14,0 \\
\hline 9 & MF3060 (59) & Fialho (RTM20-24") & 1.300 & 51 & 2,20 & 0,220 & & & & \\
\hline 10 & MF3060 (59) & Herculano (HPR20-24") & 1.300 & 46 & 2,34 & 0,160 & 610 & 150 & 240 & 17,0 \\
\hline 11 & MF3060 (59) & Herculano (HPR20-24") & 1.300 & 46 & 2,14 & 0,165 & 390 & 240 & 370 & 17,0 \\
\hline
\end{tabular}

${ }^{(1)} \mathrm{m}$ é a massa da grade; $\alpha$ é o ângulo de abertura dos corpos da grade; lé a largura de trabalho efetiva; d é a profundidade de trabalho. 
à medição do consumo específico de combustível no motor (Ce), obtida em ensaios à tomada de potência, para diferentes condições de trabalho, o que permitiu o cálculo da eficiência global na utilização do combustível. Esta eficiência reflete a relação entre a energia fornecida ao motor (combustível) e a energia utilizada pelo implemento (trabalho realizado), ou, em termos de potência, o rendimento da transformação da energia, sob a forma de combustível, fornecida ao motor por unidade de tempo (consumo horário de combustível, $\mathrm{Ch}$ ), refletese em potência na barra (produto da tração na barra pela velocidade de avanço, $\mathrm{T} \mathrm{x}_{\mathrm{r}}$ ). Este rendimento global inclui o rendimento do motor (função inversa do consumo específico), o rendimento mecânico da transmissão entre o motor e as rodas e o rendimento de tração dos pneus, em interação com o solo, e pode representar-se pela função que relaciona o consumo de combustível por hectare (Cha) com a resistência que o implemento encontra no solo, por unidade de largura de trabalho (I).

Na Figura 2 verifica-se a comparação das equações apresentadas por Bowers (1985) e Riethmuller (1989) com as retas de regressão entre o consumo de combustível por hectare e a resistência que o implemento encontra no solo por unidade de largura de trabalho, obtidas a partir dos ensaios de campo, realizados com equipamentos dos agricultores em mobilização primária de solos de textura média, para o regime de funcionamento nominal do motor e a $80 \%$ do regime de funcionamento nominal do motor. Verificou-se grande

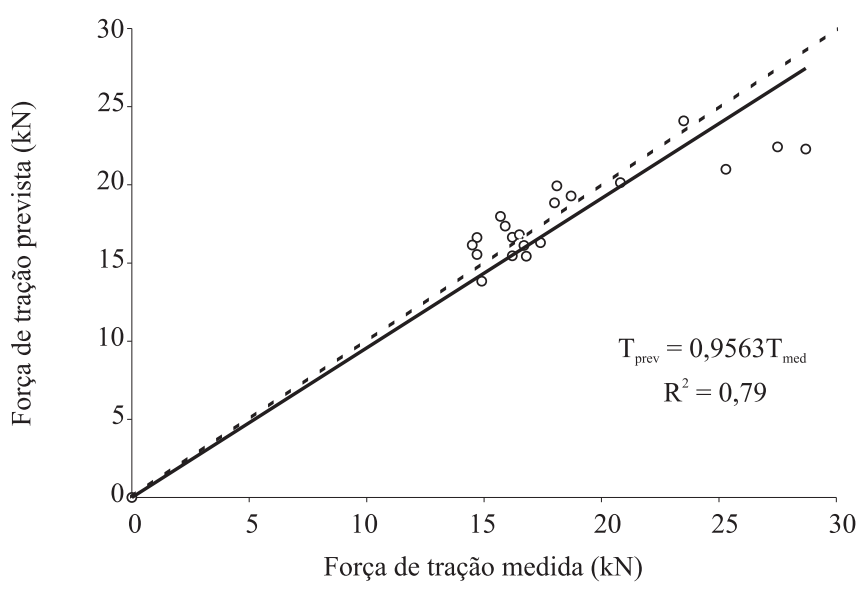

Figura 1. Enquadramento do modelo de previsão ASAE Standards (ASAE, 1995), nos valores de tração na barra, medidos nos ensaios de campo. aproximação dos modelos de previsão de Bowers (1985) e de Riethmuller (1989), respectivamente, aos valores medidos nas situações de trabalho correspondentes ao regime nominal do motor e a $80 \%$ do regime nominal do motor. Com base nesses resultados, admitindo-se que a operação cultural se realize no momento oportuno, com ajustamentos corretos no trator e no implemento, o consumo de combustível por hectare, de um trator agrícola em trabalho de preparação do solo, será determinado pelo esforço de tração requerido pela grade, refletido pela tração na barra por metro de largura do implemento.

Comprovou-se, também, o benefício de 10 a $15 \%$ no consumo de combustível, em operações de mobilização do solo, pela seleção de um regime do motor de 70 a $80 \%$ do regime nominal, relativamente à seleção do regime nominal. Esta vantagem é demonstrada pelos resultados obtidos nos ensaios à tomada de potência (Figura 3). Verifica-se que o consumo específico é um parâmetro previsível e relativamente pouco variável em certas condições de trabalho: regime semelhante de funcionamento do motor, com um grau de utilização da potência disponível acima dos $60 \%$, tal como acontece habitualmente nos trabalhos de tração. Assim, em operações de mobilização do solo, podem ser esperados os seguintes valores para o consumo específico do

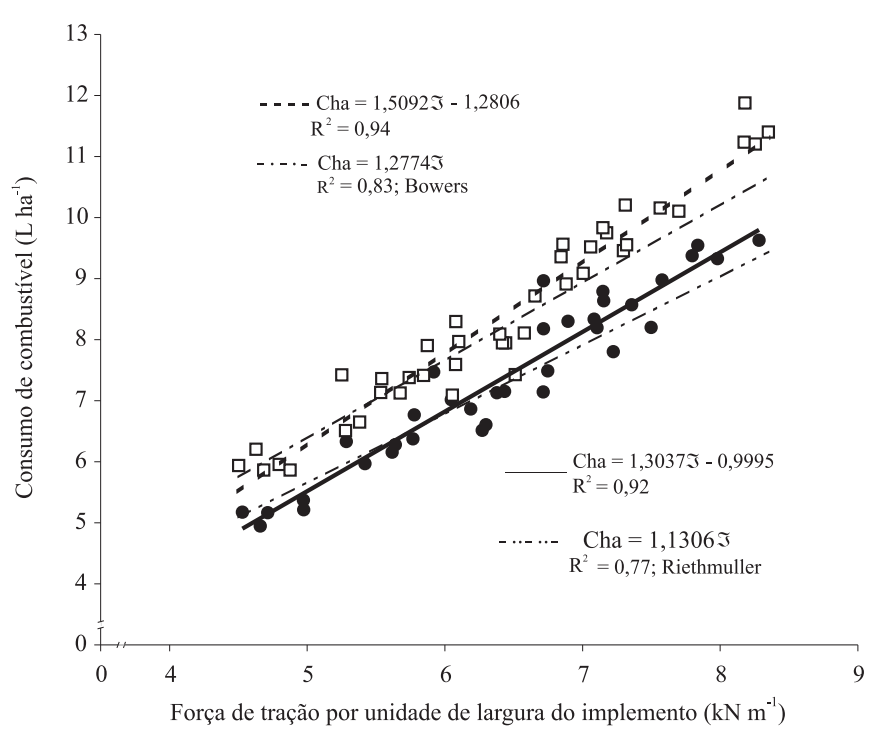

Figura 2. Previsão do consumo de combustível por hectare (Cha), a partir da força de tração na barra, por unidade de largura de trabalho do implemento (I), em dois regimes do motor: regime nominal (口) e $80 \%$ do regime nominal (•). 
motor: cerca de $265 \mathrm{~g} \mathrm{kWh}^{-1}$ em um regime de aproximadamente $80 \%$ do regime nominal, que funcione em condições que não exigem toda a potência do trator e em que se procura otimizar o consumo de combustível, sem comprometer a capacidade de trabalho $\left(\mathrm{ha} \mathrm{h}^{-1}\right.$ ); cerca de $300 \mathrm{~g} \mathrm{kWh}^{-1} \mathrm{em}$ regime nominal, utilizado em operações que exigem muita potência, ou quando se pretende otimizar a capacidade de trabalho, com uma menor eficácia em termos de consumo de combustível.

As operações de mobilização do solo representam, na utilização do trator em sistemas agrícolas tradicionais, uma das atividades com custos energéticos mais elevados. A sustentabilidade desses sistemas exige uma gestão adequada dos recursos, com redução importante dos custos de produção das culturas, principalmente em relação aos associados com o consumo de combustível. Além do correto dimensionamento do conjunto tratorimplemento, é fundamental escolher o momento adequado para intervir no solo e garantir regulagens corretas do regime do motor, da relação de transmissão da caixa de velocidades, da pressão de insuflagem dos pneus e do lastro do trator.

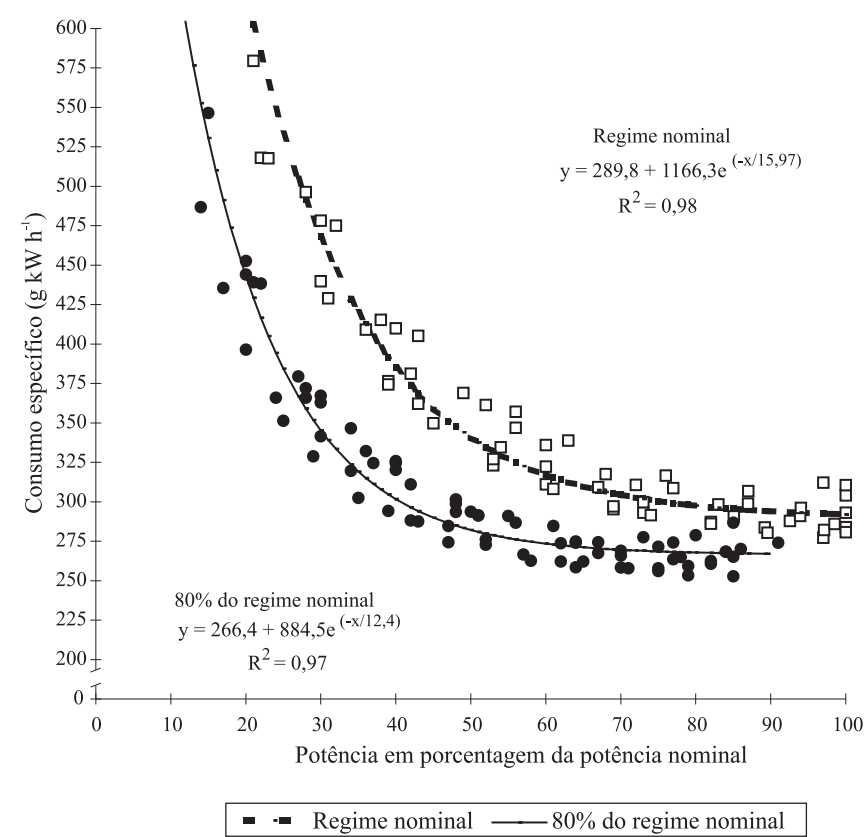

Figura 3. Consumo específico dos tratores de ensaio MasseyFerguson, em função do grau de utilização da potência nominal, em dois regimes do motor: regime nominal () e $80 \%$ do regime nominal $(\bullet)$.

\section{Conclusões}

1. Os resultados permitem estabelecer um modelo de previsão do esforço de tração requerido por grades de discos offset, a partir da massa da grade, e confirmam o modelo de previsão da American Society of Agricultural Engineers.

2. Há uma relação aproximadamente linear entre o consumo de combustível por hectare e a força de tração na barra por metro de largura de trabalho do implemento, para cada regime de funcionamento do motor e em cada condição de solo.

3. Há uma diminuição média de 10 a $15 \%$ no consumo de combustível pelo trator, na operação de mobilização do solo com grades de discos offset, pela seleção de um regime do motor de 70 a $80 \%$ do regime nominal, relativamente à seleção do regime nominal.

\section{Agradecimentos}

Ao programa Pamaf do governo português, pelo financiamento do projeto; ao Prof. J.O. Peça, do Dep. de Engenharia Rural, Universidade de Évora, pela orientação científica; à equipe do Laboratório de Medidas Elétricas do Instituto Nacional de Engenharia e Tecnologia Industrial, pelo apoio prestado na instrumentação dos tratores; à equipe de mecanização da Universidade de Trás-os-Montes e Alto Douro, pelo empréstimo do freio dinamométrico; ao pessoal do Laboratório do Dep. de Engenharia Rural, Universidade de Évora, pelo apoio prestado nas determinações de campo e análises laboratoriais; aos agricultores da região, por terem permitido a utilização dos seus equipamentos e a realização dos ensaios nas suas explorações agrícolas.

\section{Referências}

AL-JANOBI, A.A. A data-acquisition system to monitor performance of fully mounted implements. Applied Engineering in Agriculture, v.75, p.167-175, 2000.

AL-JANOBI, A.A.; AL-SUHAIBANI, S.A. Draft of primary tillage implements in sandy loam soil. Applied Engineering in Agriculture, v.14, p.343-348, 1998.

AL-SUHAIBANI, S.A.; AL-JANOBI, A.A. Draught requirements of tillage implements operating on sandy loam soil. Journal of Agricultural Engineering Research, v.66, p.177-182, 1997.

ASAE - American Society of Agricultural Engineers. ASAE D497.2: agricultural machinery management data. $42^{\text {th }}$ ed. St. Joseph: ASAE, 1995. p.335-341. 
ASAE - American Society of Agricultural Engineers. ASAE D497.4: agricultural machinery management data. $47^{\text {th }}$ ed. St. Joseph: ASAE, 2000. p.350-357.

BOWERS, C.G.J. Southeastern tillage energy data and recommended reporting. Transations of the ASAE, v.28, p.731-737, 1985.

GLANCEY, J.L.; UPADHYAYA, S.K.; CHANCELLOR, W.; RUMSEY, J.W. Prediction of agricultural implement draft using an instrumented analog tillage tool. Soil \& Tillage Research, v.37, p.47-65, 1996.

MANTOVANI, E.C.; LEPLATOIS, M.; INAMASSU, R.Y. Automação do processo de avaliação de desempenho de tratores e implementos em campo. Pesquisa Agropecuária Brasileira, v.34, p.1241-1246, 1999.

PEÇA, J.O.; SERRANO, J.M.; PINHEIRO, A.; CARVALHO, M.; NUNES, M.; RIBEIRO, L.; SANTOS, F. Tractor performance monitors optimizing tractor and implement dynamics in tillage operations - one year of field tests. In: INTERNATIONAL
CONFERENCE ON AGRICULTURAL ENGINEERING, 1., 1998. Oslo. Anais. Oslo: AgEng, 1998.

PERFECT, E.; MCLAUGHLIN, N.B.; KAY, B.D. Energy requirements for conventional tillage following different crop rotations. Transactions of the ASAE, v.40, p.45-49, 1997.

RAGNI, L.; SANTORO, G. Trattore sensibilizzato. Macchine e Motori Agricoli, v.55, p.39-45, 1997.

RIETHMULLER, G.P. Draft requirements of tillage equipment in the western Australian wheatbelt. Agricultural Engineering Australia, v.18, p.17-22, 1989.

SERRANO, J.M. Contribuição para a otimização do sistema dinâmico trator-implemento em mobilização do solo. 2002. 234p. Tese (Doutorado) - Universidade de Évora, Évora.

SERRANO, J.M.; PEÇA, J.O.; PINHEIRO, A.; CARVALHO, M.; NUNES, M.; RIBEIRO, L.; SANTOS, L. The effect of gang angle of offset disc harrows on soil tilth, work rate and fuel consumption. Biosystems Engineering, v.84, p.171-176, 2003. 